\title{
Le problème de la continuité en lexicologie historique. Réflexions à partir de la pratique lexicographique dans le cadre du projet DETCOL*
}

\author{
Marta Andronache \\ ATILF CNRS Nancy-Université \\ marta.andronache@atilf.fr
}

\section{Introduction}

Nous nous proposons de traiter le sujet de la continuité par rapport aux premières attestations à partir des principes théoriques fondamentaux formulés en lexicologie historique (Möhren 1982; Höfler 1986; Glessgen 1992 ; Städtler 2006) et de la pratique lexicographique développée dans le cadre du projet ANR DETCOL (Développement et exploitation textuelle d'un corpus d'œuvres linguistiques) dirigé par Bernard Colombat.

Le but de notre communication est de mettre en relief l'importance de la continuité comme principe lexicologique à prendre obligatoirement en compte quand le lexicologue établit la « carte d'identité » des mots. La continuité est essentielle pour donner une base scientifiquement solide et vérifiable quand il faut décider si un lexème est à dater selon la formule conventionnelle attesté depuis ou, inversement, attesté en (telle date) ou attesté de ... à .... À un niveau plus abstrait, nous serons amenée à poser la question de savoir si, dans les cas où la continuité fait défaut, il faut considérer qu'il s'agit d'unités lexicales distinctes, d'homonymes diachroniques ou sémantiques.

Notre recherche s'inscrit dans le cadre de la conception moderne de l'étymologie comme " biographie » d'un mot ( $c f$. Baldinger $1990\left[1959^{1}\right]$ ) et se rattache à la description historique des lexèmes : datation, voies de transmission, «milieu créateur » (vonWartburg [cf. Zamboni $1976: 51])$. Notre problématique rejoint par ailleurs celle des homonymes diachroniques engendrés par la dérivation ( $c f$. Chauveau 2005).

De façon sans doute un peu étonnante, cette réflexion nous amène à donner une nouvelle dimension au problème des homonymes et à repenser la définition même du terme d'antédatation puisque le problème de la continuité en lexicologie historique rejoint celui de la datation. Le terme antédatation comporte le lexème dater, verbe transitif direct qui amène implicitement la question "dater quoi ? ». Nous verrons que la réponse ne peut être que la suivante : une unité lexicale.

\section{Méthode}

Notre méthode consiste à démontrer l'importance de la question de la continuité à partir d'un corpus d'analyses étymologiques regroupées autour du champ lexical de la terminologie grammaticale.

Le déclencheur de la reconsidération de ces étymologies a été la thèse de Thomas Städtler (1988), qui apporte des antédatations pour toute une série de termes de grammaire. La base de notre étude est constituée par les quarante notices étymologiques refondues du TLFi (Trésor de la Langue française informatisé, $c f$. http://atilf.atilf.fr/tlf.htm) appartenant à la nomenclature du projet DETCOL qui sont publiées sur le site du programme de recherche TLF-Étym (http://www.atilf.fr/tlf-etym [cf. Buchi 2005]) : les adjectifs casuel $^{2}$, copulatif, déclinable, défectif, disjonctif, épicène, explétif, interrogatif, temporel ; les adjectifs-substantifs ablatif, accusatif, antécédent, aoriste, comparatif, complétif, conjonctive, déponent, dérivatif, fréquentatif, futur, pluriel; les substantifs conjonction, datif, gémination, génitif, gérondif, imparfait $^{2}$, interjection, interrogation, négation, nominatif, parfait ${ }^{2}$, passé, pluralité, plus-que-parfait, préposition, prétérit, pronom, vocatif; l'adverbe nominalement. Dans ce qui suit nous ne considérons que les cas où le rédacteur s'est posé la question de la continuité de manière explicite. 
Notre démarche sera la suivante : dégager l'importance de la continuité à partir de quatre exemples pertinents de termes de grammaire pour lesquels nous avons pu apporter une antédatation absolue : casuel $^{2}$, explétif, gémination, parfait ${ }^{2}$. Notre communication s'inscrit ainsi dans le cadre d'un premier bilan méthodologique du projet DETCOL.

Selon la méthode que nous préconisons, la citation des sources consultées, qu'elles comportent le lexème recherché ou non, est absolument nécessaire pour démontrer la (non) lexicalisation d'un terme technique de la grammaire. Par conséquent, nous proposons non seulement de vérifier et d'attester la continuité d'un lexème, mais aussi, inversement, la non continuité d'un lexème par la preuve de son absence dans les œuvres majeures de la période où on s'attendrait à le voir employé. Autant il est possible de prouver l'existence d'un mot, autant sa non-existence peut seulement être rendue probable. C'est pourquoi nos recherches reposent sur l'examen d'une riche panoplie de sources spécialisées : lexicographie historique (dictionnaires philologiques, travaux lexicographiques); dictionnaires (monolingues ou plurilingues, du Moyen Âge à nos jours); bases textuelles (Frantext, GoogleBooks); sources spécialisées (en l'occurrence, grammaires). Avec une telle masse documentaire, nous avons la certitude de ne rien laisser passer qui ait été d'usage courant dans le domaine technique qui nous intéresse, ce qui confirmera la lexicalisation ou la non lexicalisation dans le domaine des mots grammaticaux.

\section{Présentation du projet DETCOL et du programme TLF-Étym}

Le projet ANR DETCOL (Développement et exploitation textuelle d'un corpus d'œuvres linguistiques), dirigé par Bernard Colombat, représente une coopération entre trois laboratoires : L'UMR 7597 HTL «Histoire des théories linguistiques" (CNRS/Université Paris 7), le CID «Centre d'ingénierie documentaire » de l'ENS Lyon et l'ATILF (Analyse et Traitement Informatique de la Langue Française) de Nancy. Prévu sur une durée de quatre ans (2007-2010), le projet se propose (cf. http://ctlf.enslsh.fr/articles/documents/ct projet detcol.pdf) d'établir une nouvelle représentation - bâtie directement sur les textes linguistiques étudiés dans leur épaisseur textuelle - de l'histoire de la terminologie linguistique occidentale, à la fois sous l'angle conceptuel et terminologique et sous l'angle lexicologique et étymologique. Pour cela, il prend appui sur un corpus de textes linguistiques déjà décrits en ligne (cf. CTLF, http://ctlf.ens-lsh.fr).

Par ailleurs, le programme de recherche TLF-Étym (ATILF - CNRS de Nancy), qui se propose de réviser sélectivement les notices intitulées «Étymologie et Histoire » du TLF(i), représente la structure d'accueil naturelle pour les notices étymologiques refondues dans le cadre du projet DETCOL. Dans ce contexte, l'équipe travaille notamment à l'exploitation de la thèse de Thomas Städtler; en outre, il est prévu de dépouiller la première édition de La linguistique d'Abel Hovelacque (1876) ainsi que les numéros les plus anciens de la Revue critique, deux publications que l'on devine être des réservoirs de premières attestations.

\section{Prémisses théoriques}

Notre réflexion s'inscrit dans le cadre théorique de l'étymologie (gallo-)romane telle qu'elle est pratiquée actuellement par les meilleurs spécialistes du domaine, dont les prémisses théoriques remontent à la définition classique de l'étymologie comme « biographie du mot» (Baldinger 1990 [1959]) :

\footnotetext{
«L'étymologie, au sens moderne, c'est donc la biographie du mot. Sa naissance, dont s'occupait exclusivement l'ancienne étymologie, n'en est que le point de départ. [...] c'est à travers l'histoire du mot qu'on remonte aux origines. En tout cas, l'étymologie origine a besoin de la confirmation par l'histoire du mot. » (Baldinger 1990 [1959] : $46,49)$
}

Faire l'étymologie d'un mot aujourd'hui, ce n'est pas seulement trouver son origine, mais c'est aussi mettre en évidence le «milieu créateur » dans la description de la vie du mot et des mécanismes de sa transmission. Dans son manuel d'étymologie, Alberto Zamboni (1976) reprend l'importance de ces principes et de la relation entre la sociologie et l'étymologie : 


\begin{abstract}
« La struttura interna della lingua infatti non esiste a priori, bensì dal parte dell'uomo, di cui riflette l'aspetto dualistico (forma $\sim$ contenuto): è un problema reale quello di lumeggiare i rapporti tra una data lingua e una data società o cultura, come hanno fatto Wartburg, Trier, Matoré ed altri (o, nell'antropolinguistica americana, Sapir, Whorf); l'etimologia ha perciò il senso di scoprire il milieu creatore di una innovazione lessicale, per non dire del mutamento linguistico in genere, che è il problema chiave della linguistica evolutiva. » (Zamboni $1976: 51$ )
\end{abstract}

Une distinction importante est à faire dès le départ : le même étymon ne suppose pas obligatoirement la même étymologie, et ces deux termes ne doivent surtout pas être confondus. Même si deux lexèmes remontent au même étymon, leurs étymologies peuvent être différentes : par exemple, un emprunt au latin à travers une traduction d'un texte technique du $14^{\mathrm{e}}$ siècle et un emprunt au latin à travers les grammairiens du $17^{\mathrm{e}}$ siècle n'entrent pas dans la langue à travers le même « milieu créateur »: ils n'ont donc pas la même étymologie. Dans ce cas, nous sommes en présence de deux unités lexicales, des homonymes diachroniques, car il s'agit de deux emprunts faits dans le contexte de deux cultures différentes.

A partir de ces prémisses théoriques, nous nous proposons de montrer en quoi la question de la continuité, définie comme principe indispensable dans l'approche méthodologique pour établir correctement l'acte de naissance d'un lexème, est primordiale en recherche étymologique.

\title{
5 Les premières attestations absolues : un problème bien cerné en lexicologie historique
}

«La psychologie des étymologistes » (cf. Swiggers 1991) est incontestablement marquée par la recherche des premières attestations. Mais pour que cette course aux trésors reste scientifiquement fiable, elle doit nécessairement être jalonnée par des principes lexicologiques et philologiques strictement respectés ( $c f$. Möhren 1982b ; cf. Städtler 2006).

L'importance de la datation et de l'authenticité des premières attestations largement évoquée dans les travaux fondamentaux de lexicologie et de lexicographie historiques nous rendent encore plus attentifs à la diminution significative du nombre de premières attestations après les avoir soumises au critère de la vérification des sources ( $c f$. Möhren 1982), un véritable « purgatoire » lexicographique qui seul peut nous garantir la pertinence des résultats obtenus.

Pour mieux cerner ce problème, prenons quatre jugements des premières attestations :

«La datation des mots est un élément de base de la lexicologie. Les efforts de maints chercheurs visent à fournir à la lexicographie des attestations sûres, correctement interprétées et datées. C'est la recherche des premières dates qui, dans ce procédé, occupe une place primordiale. Leur importance devrait inciter les lexicographes à vérifier scrupuleusement l'authenticité de l'attestation première. » (Möhren $1982: 3$ )

«[...] selon une vieille tradition de la lexicographie historique, la plus ancienne date est toujours considérée comme la meilleure date. [...] Cet exemple [sketch] tiré de la grande quantité de nos matériaux ne montre pas seulement une fois de plus la nécessité d'un examen minutieux des datations existantes, il souligne combien il est indispensable de bannir de la lexicographie historique toutes les dates non vérifiables, qui sont un obstacle à toute recherche ultérieure de dates. » (Höfler 1986 : 424, 426)

«[...] seule la prise en compte de tous les types d'attestations charnières résout les nombreuses questions que soulève l'histoire d'un mot. Le peu d'attention prêté aux dernières attestations et aux attestations intermédiaires a pu nuire parfois à la description même des premières attestations. » (Glessgen $1992: 4: 420$ )

«En quoi consiste la traque des premières attestations? Elle a pour but d'établir la date d'apparition d'un mot dans la langue (= datation), son usage dans un contexte précis (= attestation) pourvu de sa source dûment référencée (= localisation). 


\begin{abstract}
Datation, attestation et localisation sont différents éléments d'information que le lexicologue diachronicien se doit de pourchasser avec zèle pour lever le moindre soupçon d'obscurité et d'amphibiologie. » (Steinfeld $2006: 1$ )
\end{abstract}

Le manque de vérification des sources ( $c f$. Möhren 1982), d'harmonisation de la datation des textes anciens et la méconnaissance, même involontaire, des études théoriques déployées autour de l'utilisation critique des textes dans le travail du lexicographe diachronicien génèrent des erreurs de datation ( $c f$. Städtler 2006). De même, nous pouvons ajouter que la non prise en compte de la question de la continuité peut générer des erreurs quand on établit la date de naissance d'un lexème dans la langue moderne. En effet, établir l'étymologie d'un mot est un processus complexe : fournir une première attestation doit s'accompagner d'une enquête minutieuse sur les attestations intermédiaires pour témoigner des circonstances qui ont favorisé après sa création, sa transmission et sa continuité.

\title{
6 Le piège de la non continuité : un problème à cerner
}

Qu'en est-il de la continuité entre les premières attestations absolues et les lexèmes modernes qu'elles sont censées illustrer?

Le problème de la continuité dans le cadre de la chronologie des attestations lexicales représente un des nombreux pièges qui menacent les lexicologues et lexicographes. Au moment où le lexicographe se trouve devant une première attestation, il doit se poser au moins trois questions en ce qui concerne la datation du lexème : vérifier l'authenticité de la première attestation ( $c f$. Möhren 1982), harmoniser les sources anciennes afin qu' « une seule date (ou une seule fourchette de dates) corresponde à chaque texte cité » (Städtler $2006: 1$ ) et utiliser de manière critique les sources ( $c f$. Möhren 2006). Dans ce contexte, nous voulons ajouter un quatrième critère: vérifier scrupuleusement la continuité des témoignages intermédiaires entre la première attestation et l'état actuel du lexique, car, pour citer Frédéric Duval (2006) : «Une première attestation absolue ne signifie pas que le lexème est entré dans la langue française à cette date-là » (Duval 2006 : 14). Employer, en lexicologie historique, des formules comme « attesté depuis », " attesté en », "attesté de ... à ... », « attesté en ..., puis depuis ..., ne relève pas de la variation stylistique, mais comporte des affirmations lourdes de conséquences et pleines de significations.

Il est donc absolument nécessaire de retracer la continuité d'un lexème à partir de sa première attestation et d'accompagner toute première attestation des témoignages intermédiaires qui prouvent la vie du lexème jusqu'à l'état actuel du lexique. Dans la pratique, une attestation par siècle fournira un critère de vérification de la continuité du lexème depuis son entrée dans le lexique jusqu'à nos jours, selon le principe affirmé par Max Pfister (1984) : «Se come data si indica dal..., cio significa che dopo la data registrata esiste per ogni secolo almeno un esempio e che quindi è attestata una continuità d'uso. » (Pfister $1984: 3)$

Pour aboutir à un résultat presque mathématiquement correct, le lexicographe peut appliquer l'équation de Bernard Pottier (1958) convertie au critère de la continuité des attestations : " Le nombre des formes relevées est inversement proportionnel au degré d'erreur possible » (Pottier 1958 : 581).

En conséquence, il est impératif non seulement de vérifier et d'attester la continuité d'un lexème, mais aussi d'attester sa non continuité et donc son absence dans les œuvres majeures de la période considérée.

Pour illustrer notre sujet, nous nous appuyons sur quatre exemples pertinents choisis parmi les notices refaites dans le cadre du programme de recherche TLF-Étym : deux lexèmes pour lesquels nous avons pu établir une continuité depuis la première attestation absolue (explétif et parfait) et deux lexèmes où cette continuité fait défaut (casuel et gémination). Cela nous permettra de dégager quelques principes relatifs au critère de la continuité quand on établit l'étymologie d'un lexème et à poser la question de la définition du terme antédation. 


\section{Deux exemples de continuité depuis la première attestation absolue}

\subsection{Explétif}

«Attesté depuis » traduit l'idée que le lexème est entré dans la langue (en l'occurrence française) et qu'il s'y retrouve depuis cette date jusqu'à nos jours. Dans ce cas, le lexicographe est amené à fournir des attestations suffisantes qui témoignent de la continuité du mot. Nous présenterons ci-dessous un terme de grammaire, l'adjectif explétif, pour illustrer le cas des lexèmes pour lesquels nous avons pu établir l'existence d'une chaîne ininterrompue de témoignages depuis leur première attestation absolue.

\subsubsection{Point de départ : TLF}

Dans sa partie «Étymologie et Histoire », le TLF (1980) atteste l'adjectif explétif dans la langue française depuis le $14^{\mathrm{e}}$ siècle et il le définit comme « inutile au sens ou qui n'est pas exigé par la syntaxe, mais qui sert, surtout dans la langue écrite, à colorer la phrase généralement d'une nuance affective » :

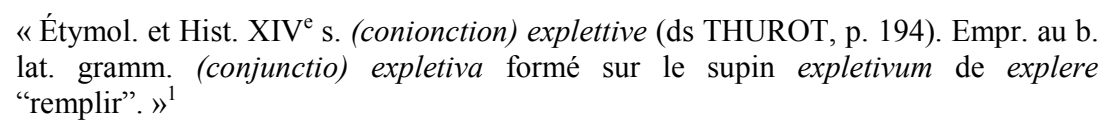

Le TLF se limite donc à donner la date de la première attestation absolue suivie d'une référence, ce qui n'est pas très satisfaisant. En effet, une première attestation signifie que le lexème est entré une première fois dans la langue française à cette date, mais cela ne signifie pas forcément qu'il est resté dans la langue française depuis cette date jusqu'à nos jours. Il faut aussi dire que dans la plupart des cas les rédacteurs du TLF n'avaient pas la possibilité de trouver des attestations pour chaque siècle à cause du peu d'occurrences qu'ils avaient à leur disposition.

\subsubsection{Antédatation proposée par Thomas Städtler}

Dans la thèse de Thomas Städtler (1988), explétif est antédaté d'un demi-siècle par rapport au TLF en se basant sur une attestation d'une traduction de l'Ars minor d'Aelius Donatus qui remonte à la $2^{\mathrm{e}}$ moitié du $13^{\mathrm{e}}$ siècle :

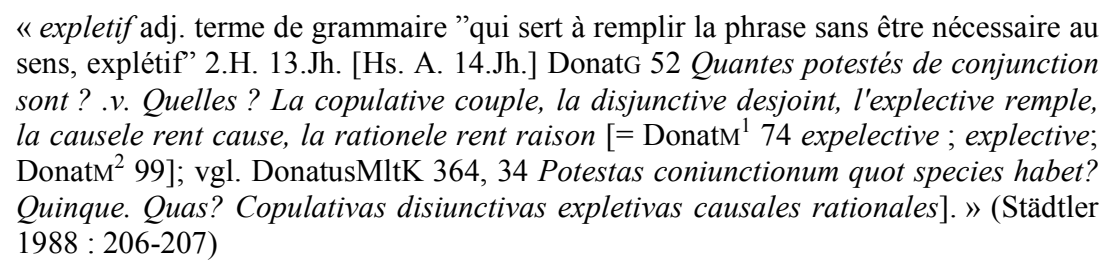

Outre cette antédatation, Thomas Städtler (1988) donne aussi un aperçu sur la lexicalisation du lexème depuis les traductions de Donat à travers les attestations du mot dans les grands dictionnaires de l'histoire de la langue française.

\subsubsection{Solution proposée par TLF-Étym}

Selon la méthode que nous préconisons, si le fait d'antédater le lexème est évidemment très important pour l'histoire du mot, il reste encore à déterminer si le mot présente véritablement une continuité depuis le moment où il apparaît pour la première fois dans notre langue jusqu'à nos jours pour pouvoir vraiment affirmer que l'usage d'explétif est attesté depuis la $2^{\mathrm{e}}$ moitié du $13^{\mathrm{e}}$ siècle.

Nous avons repris l'antédatation très précieuse fournie par Thomas Städtler (1988) tout en y apportant les témoignages de la continuité du lexème pour démontrer que l'adjectif explétif date en effet de la $2^{\mathrm{e}}$ moitié $\mathrm{du} 13^{\mathrm{e}}$ siècle, vu qu'il est attesté sans interruption du Moyen Âge jusqu'à nos jours. Nous avons pu ainsi 
retrouver des attestations pour chaque siècle et nous avons doublé ces indications d'une précision sur sa lexicographisation :
«Adj. „qui est usité dans une phrase sans être nécessaire pour la compréhension du sens (terme de grammaire)“. Attesté depuis la $2^{\mathrm{e}}$ moitié $13^{\mathrm{e}}$ siècle (DonatgS, page 95 , $\S 52$ : Quantes potestés de conjunction sont? .v. Quelles? La copulative couple, la disjunctive desjoint, l'explective remple, la causele rent cause, la rationele rent raison). En plus des attestations tirées de Donat (début $14^{\mathrm{e}}$ siècle ; $c a 1325=\underline{\text { Thurot, }}$ $\underline{\text { Notices, }}$ page $194=\underline{\text { TL }}$; Städtler, Grammatiksprache 206-207), le mot est attesté sans interruption notamment dans des glossaires latin-français à partir de la $2^{\mathrm{e}}$ moitié du $14^{\mathrm{e}}$ siècle (AalmaR, page $129, \mathrm{n}^{\circ} 3.718 ; \mathrm{ca} 1420 / 1440$, LeVerM, page 158, s.v. expleo ; 1467/1506, MolinetFaictzD, volume 2, page 692, vers $317=\underline{\mathrm{DMF} 2}$; fin $15^{\mathrm{e}}$ siècle, LeTallE, page $113 ; 1499, \underline{\text { GlLag1499}}$, page 71, cf. $\underline{\text { GdfC }) . ~[\ldots] ~} »^{2}$

Dans le cas de l'adjectif explétif la première attestation absolue, de la $2^{\mathrm{e}}$ moitié $13^{\mathrm{e}}$ siècle, représente donc un début de lexicalisation réussie puisque l'adjectif explétif s'est imposé et a survécu jusque dans l'état actuel de la langue contemporaine. Sa continuité prouve que nous sommes aujourd'hui devant la même unité lexicale qu'au Moyen Âge.

\title{
7.2 Parfait
}

Dans notre pratique lexicographique il nous arrive de devoir traiter deux unités lexicales dans un même article. C'est le cas, dans le cadre du programme TLF-Étym, de l'article parfait ${ }^{2}$, qui aborde deux termes techniques de la grammaire, d'ailleurs synonymes, parfait et prétérit parfait. Ces deux unités lexicales ont une histoire différente, mais elles sont toutes les deux attestées en continu depuis leurs premiers témoignages.

\subsubsection{Point de départ : TLF}

Le TLF (1986) atteste le substantif masculin parfait avec le sémantisme « catégorie verbale présentant le procès comme entièrement accompli, et l'envisageant dans son résultat actuel » depuis 1596, dans l'introduction grammaticale du dictionnaire français-allemand de Hulsius. Cette datation est précédée par une mention de prétérit parfait au $14^{\mathrm{e}}$ siècle :

\begin{abstract}
«Étymol. et Hist. XIV s. gramm. adj. prétèrit parfait (Ms. Fonds St Victor 867. Doctrinal avec glose, Anonyme du XIV's. d'apr. Ch. THUROT ds Notices et Extraits des mss de la bibl. impériale et autres bibl., XXII, 2, p.184); 1596 gramm. subst. «temps qui marque un passé accompli» (HULSIUS, introd.). Représente la trad., par la forme parfait, du lat. perfectum tempus ou, plus brièvement, perfectum p. subst. de l'adj. perfectus, terme de gramm. du lat. »
\end{abstract}

\subsubsection{Antédatation proposée par Thomas Städtler}

S.v. parfait Thomas Städtler (1988 : 250) renvoie lui aussi à prétérit parfait. Il en fournit une première attestation de la $2^{\mathrm{e}}$ moitié du $13^{\mathrm{e}}$ siècle et apporte des données intermédiaires des $14^{\mathrm{e}}$ et $16^{\mathrm{e}}$ siècles qui témoignent de la continuité de ses attestations :

«preterit parfait $\mathrm{m}$. terme de grammaire "temps du verbe qui exprime un fait passé relativement au moment de la parole, parfait" 2.H. 13.Jh. [Hs. A. 14.A.] DonatG 34 Quans temps sont formés en la declineson du verbe? .v. Quiex? Le present, si come lego, le preterit imparfet, si come legabam, le preterit parfet ${ }^{l}$, si come legi, le preterit plus que parfet, si come legeram, le futur, si come legam [...]. Fehlt in TL, Gdf und FEW 9,322b sub PRAETERIRE; dort 8,238a sub PERFECTUS: „Die lateinischen grammatiker hatten die ausdrücke perfectum tempus und plus quam perfectum tempus gebildet, und daraus die subst. perfectum und plusquamperfectum abgezogen. Als im 16.jh. im schatten der lateinischen grammatik die französische entstand, wurden diese beiden ausdrücke, unter anlehnung an fr. parfait, übernommen, daher $\mathrm{b} \alpha[\mathrm{mfr}$. nfr. 
parfait "temps qui marque un passé accompli"], $\beta$ [temps plus que parfait etc.].“; TLF 12, 993a sub PARFAIT ${ }^{2}$ subst. masc.: XIV ${ }^{\mathrm{e}}$ s. gramm. adj. prétèrit parfait (Ms. Fonds St Victor 867. Doctrinal avec glose, Anonyme du XIV ${ }^{\mathrm{e}}$ s. d'apr. Ch. Thurot ds Notices et Extraits des mss. de la bibl. impériale et autres bibl., XXII, 2, p.184 [= ThurotEx 184, dort jedoch Donatm ${ }^{1}$ und Donatm ${ }^{2}$ !]. » (Städtler $1988: 270$ )

En outre, dans son étude «Témoins précoces de la terminologie grammaticale », Thomas Städtler (1999) fournit pour prétérit parfait une antédatation par rapport à sa thèse (1988), tirée de la grammaire latine d'Aelfric, texte qui date de la fin du $12^{\mathrm{e}}$ siècle :

\begin{abstract}
«- preterit parfait $\mathrm{m}$. terme de grammaire « temps du verbe qui exprime un fait passé par rapport au moment de la parole; parfait» fin $12^{\mathrm{e}}$ s. AelfricFH 102 tempore preterito perfecto: par le preterit parfet, et plusquamperfecto: et plusqueparfet. Emprunté au lt. praeteritum perfectum. Seule attestation avant DontaGS $34\left(2^{\mathrm{e}} \mathrm{m} \cdot 13^{\mathrm{e}}\right.$ s. [ms. déb. 14 $\mathrm{e}$ s.]), $c f$. StädtlerGram 270. » (Städtler 1999 : 128)
\end{abstract}

\title{
7.2.3 Solution proposée par TLF-Étym
}

La réponse à la question de la tradition ininterrompue ou non du terme de grammaire parfait a été apportée par Éva Buchi (TLF-Étym, 2007) : l'article parfait ${ }^{2}$ révisé fait une nette différence entre les deux termes réunis par le TLF : parfait et prétérit parfait. Il s'agit de deux unités lexicales qui présentent toutes les deux une série ininterrompue d'attestations, et dont l'histoire est intimement liée : tandis que la locution nominale prétérit parfait est attestée en continu du $12^{\mathrm{e}}$ siècle jusqu'en 1775 , le substantif parfait vient la concurrencer seulement à la fin du $16^{\mathrm{e}}$ siècle, pour l'évincer totalement au $19^{\mathrm{e}}$ siècle.

\begin{abstract}
«C. prétérit parfait loc. nom. masc. «temps verbal présentant le procès comme accompli et l'envisageant dans son résultat actuel ». Attesté de la fin $\mathrm{du} 12^{\mathrm{e}}$ siècle [par référence à la grammaire latine] (AelfricfH , page 102, in Städtler, TraLiPhi 37, page 128: tempore preterito perfecto: par le preterit parfet, et plusquamperfecto : et plusqueparfet) à 1878 (Ac7 s.v. parfait: En Grammaire, Prétérit parfait, ou substantivement, Parfait, Le prétérit qui marque une chose parfaite, une chose arrivée dans un temps qui n'est ni précis ni déterminé, comme $J^{\prime}$ ai aimé, j'ai dit [...]. L'emploi de Parfait [...] comme substantif[s] est le plus ordinaire). Cf. Städtler, Grammatiksprache, page 270 pour des attestations des $13^{\mathrm{e}}-15^{\mathrm{e}}$ siècles. Dernière attestation textuelle : 1775 (Condillac, Cours d'étude, volume 1, partie 2, chapitre 10, page 196, in Gallica $=$ Frantext : On appelle je ferois, prétérit imparfait; je fis \& j'ai [fait], prétérit parfait ; \& j'avois fait, plusque parfait). »
\end{abstract}

Pour ce qui est du simple parfait, on le relève une première fois en 1596, dans le dictionnaire allemandfrançais de Hulsius, attestation qui témoigne d'un processus de lexicalisation déjà bien entamé. Pourtant, en 1834, le volet grammatical de l'Encyclopédie Méthodique donne parfait dans un contexte qui fait encore allusion a prétérit parfait : «On dit en termes de grammaire, le Prétérit parfait, ou simplement le Parfait [...] », ce qui montre que l'indépendance du substantif parfait n'est pas encore entièrement acquise. Malgré cela, le terme est attesté sans interruption à partir de 1596 ; cette première attestation ayant été relevée dans un dictionnaire, il est toutefois fort probable que le terme était déjà employé avant cette date :

«A./B. parfait subst. masc. «temps verbal présentant le procès comme accompli et l'envisageant dans son résultat actuel ». Attesté depuis 1596 (Hulsius1, introduction grammaticale : Des Verbes [...] Present. Die zeit die jetze da ist / als : Ie mange, ich $\mathrm{e}$ ß. / Imparfait. Zeit die halb fürüber ist / Ie mangeoye, ich aß / Indiffinit. Zeit so auch fast fürüber / Ie mangeay, ich aß / Parfait. Zeit die gar fürüber ist / I'ai mãgé, ich hab gessen. / Advenir. Zeit so noch zukünftig ist / Ie mangeray, ich werde essen). Première attestation dans une grammaire française : 1606 (Masset, Acheminement, page 11: Nos verbes ont cinq modes. Indicatif, imperatif, optatif, subiunctif, \& infinitif: Trois temps principaux, present, passé \& futur. Le passé se diuise en imparfait, aoriste simple, parfait, plus que parfait, aoriste composé, \& parfait, tres-parfait ; $c f$. aussi Maupas, Grammaire2, page $92 \mathrm{v}^{\mathrm{o}} / 93 \mathrm{v}^{\mathrm{o}}$ ). » 
Nous pouvons constater que prétérit parfait et parfait sont intimement liés par le fait même d'appartenir à la même famille de mots. Par contre, l'origine de parfait est bien distincte de celle de prétérit parfait: tandis que la locution nominale représente un calque du latin praeteritum perfectum, le substantif parfait est une formation française issue par ellipse de prétérit parfait:

\begin{abstract}
« Origine
C. Transfert linguistique : calque du latin praeteritum perfectum loc. nom. neutre « temps verbal présentant le procès comme accompli et l'envisageant dans son résultat actuel (terme de grammaire)» (attesté depuis Quintilien, TLL 10/1, 1378, s.v.

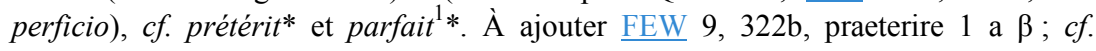
Städtler, Grammatiksprache 270.

A./B. Formation française : ellipse de prétérit parfait (cf. ci-dessus C.). Cette analyse se recommande en raison des témoignages explicites des sources lexicographiques ( $c f$. "ou substantivement" dans la citation ci-dessus C.) ainsi que du parallélisme d'imparfait (< prétérit imparfait, cf. imparfait* et prétérit*). Cf. von Wartburg in FEW $8,237 \mathrm{~b}$, perrěctus I $1 \mathrm{~b} \alpha$, qui avance à tort l'hypothèse d'un calque du latin perfectum subst. neutre «temps verbal présentant le procès comme accompli et l'envisageant dans son résultat actuel (terme de grammaire) » (attesté depuis Varron, TLL 10/1, 1377, s.v. perficio) sur parfait ${ }^{1}$. On écarte de même l'hypothèse d'un emprunt à l'allemand Perfekt subst. neutre « temps verbal présentant le procès comme accompli et l'envisageant dans son résultat actuel» (attesté depuis le $17^{\mathrm{e}} / 18^{\mathrm{e}}$ siècle seulement, Schulz, Fremdwörterbuch1), même si la première attestation absolue se trouve dans un dictionnaire allemand-français.

Dès les premiers témoignages d'un discours grammatical français, on relève le latinisme prétérit parfait (ci-dessus C.). C'est seulement à la toute fin du $16^{\mathrm{e}}$ siècle (en 1550, Meigret, Traité, page 70 emploie encore prétérit parfait) que parfait (ci-dessus A./B.) vient concurrencer la locution nominale, avant de l'évincer complètement au $19^{\mathrm{e}}$ siècle. - L'adjectif parfait à sens grammatical ( $c f$. Städtler, Grammatiksprache 250-251) n'a pas de rapport étymologique direct avec le lexème traité ici ; il serait à classer s.v. parfait ${ }^{1 *}$. »
\end{abstract}

\title{
8 Deux exemples de non continuité depuis la première attestation absolue
}

\subsection{Casuel}

En revanche, nous rencontrons souvent des lexèmes pour lesquels il n'existe pas de continuité entre une première attestation isolée et la langue contemporaine. Il s'agit des cas où la lexicalisation a échoué dans un premier temps : un mot est attesté une première fois de manière isolée, suivi par de longs siècles sans autres attestations. Il arrive très souvent que ce type de lexème fasse sa réentrée dans la langue tardivement par rapport à la première attestation isolée. C'est le cas par exemple d'un autre terme de grammaire, l'adjectif casuel $^{2}$. Dans ce cas, il faut faire la distinction entre la première tentative de lexicalisation et le moment où le mot est réellement entré dans le lexique français.

\subsubsection{Point de départ : TLF}

Dans le TLF (1977), l'adjectif casuel relevé avec le sémantisme « qui comporte des cas grammaticaux, ou se rapporte à eux » est attesté dans la langue française depuis le milieu du $19^{\mathrm{e}}$ siècle en s'appuyant sur un renvoi du Larousse (1866) à une œuvre non précisée d'Ernest Renan :

$$
\begin{aligned}
& \text { «Étymol. et Hist. Mil. XIX }{ }^{\mathrm{e}} \text { s. (Renan ds Lar. } 19^{e} \text { ). Empr. au lat. casualis, terme de } \\
& \text { gramm. au sens de «relatif au cas » attesté dep. Varron (TLL s.v., 571, 54).» }
\end{aligned}
$$

Le rédacteur de la partie diachronique de l'article du TLF ne s'est pas totalement trompé quand il a daté si tardivement ce mot, mais des recherches plus approfondies nous ont permis de retracer l'histoire de ce terme de grammaire avec plus de précision. 


\title{
8.1.2 Antédatation proposée par Thomas Städtler
}

Dans sa thèse, Thomas Städtler (1988) fournit pour l'adjectif casuel une antédatation de cinq siècles par rapport au TLF en se basant sur une attestation d'une traduction de la grammaire latine de Donat dont le texte date de circa 1325 :

\begin{abstract}
«casuel adj. terme de grammaire „qui est objet des flexions d'une déclinaison“ ca.1325 Donatm ${ }^{2} 42$ Quantes fourmes casueles sont? .vij. Queles? Monoptota, diptota, triptota, tetratota, penthatota, exaptota, aptota [vgl. DonatusArsK 377,23 sunt autem formae casuales sex, ex quibus sunt nomina alia monoptota, alia diptota, alia triptota, alia tetraptota, alia pantaptota, alia hexaptota. Sunt praeterhaec aptota, quae neque per casus neque per numeros declinatur] » (Städtler $1988: 174)$
\end{abstract}

En plus de cette antédatation spectaculaire, Thomas Städtler (1988) donne des attestations du mot au $15^{\mathrm{e}}$ siècle dans des traités de grammaire du latin.

\subsubsection{Solution proposée par TLF-Étym}

Pourtant, cette première attestation n'entraîne pas automatiquement l'affirmation : le lexème casuel date de $c a 1325$. Même si le mot est attesté aussi au $15^{\mathrm{e}}$ siècle, il reste à fournir les attestations intermédiaires qui témoignent de sa continuité depuis cette date jusqu'au milieu du $19^{\mathrm{e}}$ siècle quand l'adjectif casuel est de nouveau attesté dans le lexique français.

Nos recherches, menées conjointement avec Nadine Steinfeld, ont révélé une absence totale d'attestations intermédiaires entre les attestations du Moyen Âge et celles du $19^{\mathrm{e}}$ siècle. Les premières attestations sont incontestablement très importantes, mais elles ne prouvent pas à elles seules que l'adjectif casuel date de ca 1325 dans le lexique français moderne. Dans ce cas, nous sommes en présence d'homonymes diachroniques et nous avons donc deux unités lexicales distinctes : il s'agit d'un premier emprunt de l'adjectif casuel au Moyen Âge et d'un réemprunt au $19^{\mathrm{e}}$ siècle :

\begin{abstract}
«Attesté depuis 1863 (Renan, Système, page 402, § 8: „Enfin l'ancienneté des flexions casuelles a reçu, dans ces dernières années, une confirmation inattendue du déchiffrement des inscriptions qu'on lit sur les rochers du mont Sinai““). Le mot semble être définitivement entré dans la terminologie linguistique en 1876 à travers l'œuvre de Hovelacque (Hovelacque, Linguistique1, page 163: „Pour M. Frédéric Müller les désinences casuelles auraient été : $\hat{u}$, pour hâu pronom de la troisième personne; $\hat{\imath}$, suffixe indiquant la relation; le démonstratif $\left.a n^{\prime \prime}\right)$. On relève une première tentative de lexicalisation de casuel dans un sens grammatical (Städtler, Grammatiksprache, page 174) de ca. 1325 (Donatm2S, page 115, § 42 : „Quantes fourmes casueles sont? [...] Queles ? Monoptota, diptota, triptota, tetra[p]tota, pentha[p]tota, exaptota, aptota") et au $15^{\mathrm{e}}$ siècle (Gramm5S, page $149, \S 13$ : „assçavoir est que quant pluseures dictions casueles concourrent en une meisme oroison soit mediate soit immediate, et samble qu'elles appartiengnent a une meisme chose, ellez doibvent estre d'ung meisme case, si come le bon preux homme, maistre Piere, nostre curé, chemine droit et reid"). Les grammaires du $16^{\mathrm{e}}$ siècle (Ø Estienne, Traicté ; Ø Meigret, Traité ; Ø La Ramée, Grammaire), ainsi que du $18^{\mathrm{e}}$ siècle (Ø Encyclopédie Méthodique, Grammaire) n'utilisent pas le terme casuel. Cela fait apparaître qu'il n'y a pas de continuité entre les attestations du Moyen Âge et le terme appartenant au lexique actuel. Nous n'avons pas trouvé d'attestation non plus dans les grands dictionnaires du $17^{\mathrm{e}}$ siècle (Ø $\underline{\text { Cotgrave }} ; \varnothing \underline{\text { Furetière1 })}$. La première attestation lexicographique date de 1867 (Larousse1: „Gramm. Qui se rapporte aux cas, aux désinences variables des mots déclinables: En arabe, les flexions casuelles s'expriment par des lettres quiescentes [Renan]"). »
\end{abstract}

Conformément à la méthode que nous proposons, nous avons donc vérifié l'absence de ce lexème aux $16^{\mathrm{e}}, 17^{\mathrm{e}}$ et $18^{\mathrm{e}}$ siècles dans les grammaires les plus importantes (Estienne, Meigret, La Ramée, Maupas, Encyclopédie Méthodique Grammaire) et dans les dictionnaires marquants de cette période (Cotgrave, Furetière, Richelet, Académie). 
Il faut remarquer que «le milieu créateur » de l'adjectif casuel est différent selon les époques auxquelles il est emprunté : au Moyen Âge il s'agit d'un emprunt limité apparemment aux traductions de Donat, grammairien latin du $4^{\mathrm{e}}$ siècle, tandis qu'au $19^{\mathrm{e}}$ siècle le mot est réemprunté à travers des ouvrages de linguistique dans un contexte culturel tout à fait distinct.

\subsection{Gémination}

Le cas de la non continuité est encore plus limpide quand on constate un sémantisme divergent : prenons l'exemple du substantif féminin gémination « redoublement, dans l'écriture ou la prononciation, d'une voyelle, d'une consonne (et plus rarement d'une syllabe) ». Le mot est attesté dans le lexique actuel depuis 1905 même si nous avons une première attestation isolée qui date de 1325. L'analyse philologique des sources a montré que nous sommes en présence de deux unités lexicales différentes, car il s'agit d'homonymes sémantiques.

\subsubsection{Point de départ : TLF}

Le TLF (1981) propose une seule et unique origine pour les quatre sens du substantif féminin gémination (en botanique, chirurgie dentaire, enseignement et linguistique):

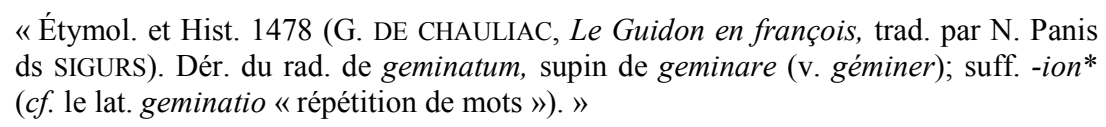

Le rédacteur de la partie diachronique de l'article du TLF ne s'est pas trompé quand il a constaté que le mot est attesté depuis le $15^{\mathrm{e}}$ siècle, mais cette datation n'est valable que pour le sens chirurgical. Des recherches plus approfondies ont permis d'établir aussi l'origine et l'histoire du terme grammatical.

\subsubsection{Antédatation proposée par Thomas Städtler}

Dans sa thèse, Thomas Städtler (1988) fournit pour le substantif féminin gémination une première attestation absolue dans la traduction de la grammaire latine de Donat dont le texte date de circa 1325 :

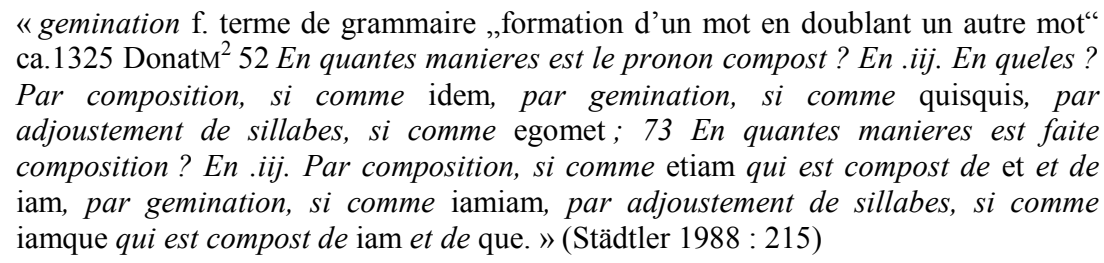

Dans ses commentaires, Thomas Städtler (1988) donne des précisions sur les différents sémantismes de ce mot retenus par la lexicographie des $19^{\mathrm{e}}$ et $20^{\mathrm{e}}$ siècles, ce qui nous met déjà sur la piste de la non continuité du sens grammatical de gémination entre cette première attestation du $14^{\mathrm{e}}$ siècle et le lexème appartenant au français moderne.

\subsubsection{Solution proposée par TLF-Étym}

Tout comme dans le cas de l'adjectif casuel, cette première attestation n'entraîne pas automatiquement l'affirmation que le lexème daterait de $c a$ 1325. L'analyse entreprise par Tatiana Kameneva à travers un nombre important d'œuvres grammaticales et lexicographiques représentatives fait apparaître que le lexème gémination avec un sens grammatical est totalement absent entre le $14^{\mathrm{e}}$ et $1 \mathrm{e} 20^{\mathrm{e}}$ siècle, quand le mot fait à nouveau son apparition dans le lexique français.

Cet écart de cinq siècles entre la seule attestation du Moyen Âge et le substantif gémination appartenant au lexique actuel prouve qu'il s'agit encore une fois d'homonymes diachroniques, et nous sommes donc 
en présence de deux unités lexicales distinctes : un premier emprunt ponctuel du substantif gémination comme terme de grammaire a été fait au Moyen Âge et il a été réemprunté pour de bon au $20^{\mathrm{e}}$ siècle :

\begin{abstract}
«D. «redoublement, dans l'écriture ou la prononciation, d'une voyelle, d'une consonne (et plus rarement d'une syllabe) ». Attesté depuis 1905 (Meillet, Abrégé, page 37, in DDL 39 : À cause de l'impression double qu'en reçoit l'oreille, on nomme aussi gémination cette division de la consonne). On relève une toute première attestation isolée de gémination dans un sens grammatical dès le Moyen Âge, mais le sens y est «type de composition qui consiste à répéter deux fois la même unité lexicale pour en former une nouvelle»: 1325 (Donatm2S, page 121, § 73: En quantes manieres est faite composition? En .iij. Par composition, si comme etiam qui est compost de et de iam, par gemination, si comme iamiam, par adjoustement de sillabes, si comme iamque qui est compost de iam et de que). L'analyse de nombreux ouvrages de grammaire embrassant la période du $16^{\mathrm{e}}$ au premier quart du $20^{\mathrm{e}}$ siècle $(\varnothing$

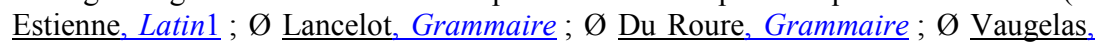
Remarques; $\varnothing$ Antonini, Principes ; $\varnothing$ Encyclopédie Méthodique, Grammaire; $\varnothing$ Dictionnaire Conversation Lecture; $\varnothing$ Portius ; Grammatica, éd. Meyer-Lübke; $\varnothing$

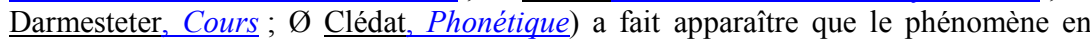
question est décrit sous le nom de dédoublement, redoublement ou réduplication, mais jamais sous celui de gémination. Par conséquent, il n'y a aucune continuité entre l'attestation isolée du $14^{\mathrm{e}}$ siècle et le terme technique moderne. »
\end{abstract}

Conformément à notre méthode, nous avons donc vérifié l'absence de ce lexème entre le $16^{\mathrm{e}}$ et le $20^{\mathrm{e}}$ siècle dans les grammaires représentatives (Estienne, Meigret, La Ramée, Vaugelas, Encyclopédie Méthodique Grammaire, Darmesteter, Clédat) et dans les dictionnaires marquants de cette période (Cotgrave, Furetière, Richelet, Académie).

Le « milieu créateur » du premier emprunt de gémination est à chercher du côté du traducteur de Donat, tandis qu'au $20^{\mathrm{e}}$ siècle le mot est réemprunté dans un contexte culturel bien distinct, puisqu'il apparaît d'abord dans un ouvrage de grammaire comparée des langues indo-européennes.

\title{
9 Résultats
}

Dans ce qui précède, nous espérons avoir démontré que dans le cas des termes de grammaire explétif et parfait, il s'agit de lexèmes qui grâce à des attestations régulières prouvent leur continuité depuis le témoignage le plus ancien. En revanche, pour casuel et gémination, nous avons établi leur non continuité. Dans le cas de gémination nous sommes en présence d'homonymes diachroniques. Même dans les cas où l'étymologie est semblable, si le fil de la continuité est rompu nous sommes en présence de deux unités lexicales distinctes qui se caractérisent par un «milieu créateur » différent et qui se trouvent dans une relation d'homonymie diachronique entre elles.

Ces résultats ne s'appuient pas seulement sur ces quatre exemples, mais sur les quarante notices étymologiques refaites dans le cadre du programme de recherche TLF-Étym et du projet ANR DETCOL. Les lexèmes présents en continu depuis leurs premières attestations absolues sont les plus nombreux puisqu'ils représentent $85 \%$ des cas : ablatif, accusatif, antécédent, aoriste, comparatif, conjonctive, conjonction, copulatif, datif, déponent, dérivatif, disjonctif, épicène, explétif, fréquentatif, futur, génitif, gérondif, imparfait ${ }^{2}$, interjection, interrogatif, interrogation, négation, nominatif, parfait ${ }^{2}$, passé, pluralité, pluriel, plus-que-parfait, préposition, prétérit, pronom, temporel, vocatif. Pour les autres $15 \%$ des cas les premières attestations restent isolées et ne marquent qu'une tentative de lexicalisation manquée. Il s'agit de casuel $^{2}$, complétif, déclinable, défectif, gémination et nominalement.

Par conséquent, le mot attesté isolément est une unité lexicale différente du mot qui fait partie de la langue contemporaine. Ce dernier est à dater depuis la première attestation présentant de la continuité avec l'emploi actuel du mot. 
Il faut dire que les mots grammaticaux appartiennent au vocabulaire technique et qu'ils représentent un aspect particulier du lexique. Ainsi on constate que certains termes de ce champ lexical sont très peu fréquents ou bien sont concurrencés par des homonymes diachroniques.

Bien sûr, l'établissement de la (non) continuité dans l'historique de ces lexèmes est tributaire de l'état actuel des dépouillements textuels, qui n'est pas parfait. Mais l'éventail des sources utilisées est suffisamment vaste pour qu'on ait l'assurance que les unités lexicales qui n'y apparaissent pas n'étaient, pour le moins, pas d'usage courant. En outre la panoplie des sources spécialisées pour les termes grammaticaux que se propose de réunir le projet DETCOL permettra de vérifier la continuité ou la non continuité des termes en cause dans le discours des grammairiens et linguistes.

\section{Conclusion}

Nous considérons que la continuité doit être prise en compte parmi les grands principes de la lexicologie historique moderne. À partir de maintenant le lexicologue diachronicien ne pourra plus ignorer ce principe, puisque nous avons pu voir que la continuité est essentielle pour une datation et une description historique correcte des lexèmes. Trouver des premières attestations est sans doute primordial, mais cette démarche doit se compléter par une attention toute particulière aux attestations intermédiaires qui témoignent de la continuité interne. Une première attestation d'un lexème qui ne se trouve pas dans un rapport de continuité avec l'unité lexicale à expliquer n'est pas pertinente pour l'histoire de cette unité lexicale. Dans ce cas il est faux de faire remonter l'apparition d'un lexème dans la langue moderne à sa première attestation isolée.

En effet, s'il n'y pas de continuité entre le lexème attesté isolément et le lexème qui fait partie du lexique français contemporain il s'agit de deux unités lexicales distinctes qui se caractérisent par un «milieu créateur » différent et qui se trouvent dans une relation d'homonymie diachronique entre elles. Par conséquent, il est important non seulement d'apporter des témoignages de la continuité d'un lexème, mais aussi de mettre en évidence la non continuité et donc l'absence d'un lexème des ouvrages marquants de la période considérée.

Ces considérations nous amènent à nous poser la question suivante, que nous soumettons à la sagacité de la communauté scientifique : ne devrait-on pas modifier la définition du terme technique d'antédatation, en réservant ce terme aux cas présentant une continuité (comme explétif ou parfait), puisqu'on antédate une même unité lexicale, et parler pour les autres cas, comme casuel ou gémination, de premières attestations isolées ou d'antéattestations?

\section{Références bibliographiques}

Baldinger, K. (1990 [1959]). L'Etymologie hier et aujourd'hui, Die Faszination der Sprachwissenschaft, Tübingen : Niemeyer, 40-73.

Buchi, E. (2005). Le projet TLF-Étym (projet de révision sélective des notices étymologiques du Trésor de la langue française informatisé), Estudis romànics, 27, 569 - 571.

Chauveau, J.-P. (2005). Remarques sur la dérivation dans les notices historiques et étymologiques du Trésor de la langue française, Actes du Séminaire de méthodologie en étymologie et histoire du lexique, Nancy: ATILF, (CNRS/Université Nancy 2/UHP), (http://www.atilf.fr/atilf/seminaires/Seminaire Chauveau 2005-11.pdf.)

Clédat, L. (1917). Manuel de Phonétique et Morphologie Historique du Français, Paris : Hachette.

Cotgrave, R. (1950 [1611]). A Dictionarie of the French and English Tongues, édité par William S. Woods, Columbia : University of South Carolina Press.

Darmesteter, A. (1891-1897). Cours de grammaire historique de la langue française, 4 volumes, édités par Ernest Muret et Léopold Sudre, Paris : Delagrave.

Donat [traduction de l'Ars minor d'Aegilius Donatus]. (1988 [ ca 1325]). In : Thomas Städtler, Zu den Anfängen der französischen Grammatiksprache. Textausgaben und Wortschatzstudien, Tübingen : Niemeyer, 109-125. 
Duval, F. (2006). Pour la révision des mots de civilisation romaine du Trésor de la langue française (informatisé), Actes du Séminaire de méthodologie en étymologie et histoire du lexique, Nancy: ATILF, (CNRS/Université Nancy 2/UHP), (http://www.atilf.fr/atilf/seminaires/Seminaire Duval 2006-11.pdf).

Encyclopédie Méthodique, Grammaire = Beauzée, N. de/Marmontel, J.-F. (1789 [1782/1784/1786]). Dictionnaire de grammaire et de littérature suivi d'un double tableau d'analyse. Extrait de l'Encyclopédie méthodique, 6 volumes, Liège : Société typographique.

Estienne, R. (2003 [1557]). Traicté de la Grammaire Françoise (1557), édité par Colette Demaizière, Paris: Champion.

Furetière, A. (1690). Dictionaire universel contenant generalement tous les mots françois tant vieux que modernes, les Termes de toutes les sciences et des arts, 2 volumes, La Haye-Rotterdam : A.-R. Leers.

Glessgen, M. (1992). Qu'est-ce qu'une attestation charnière? Quelques considérations sur le traitement du vocabulaire scientifique médiéval dans les dictionnaires étymologiques italiens, Actes du XX Congrès International de Linguistique et de Philologie Romane, Tübingen/Bâle : Francke, 4, 420-432.

Höfler, M. (1986). Typologie des erreurs de datation dans la lexicographie française, Revue de linguistique romane, $50,423-441$.

Hulsius, (Levinus Hulst, dit). (1992 [1596 $\left.\left.{ }^{1}\right]\right)$. Dictionnaire françois-alemand et alemand-françois, édité par Laurent Bray, Erlangen, Fischer.

La Ramée, P. (2001 [1572] ]). Grammaire (1572), édité par Colette Demaizière, Paris : Champion.

Maupas, Ch. $\left(1632^{5}\left[1607^{1}\right]\right)$. Grammaire et syntaxe francoise contenant reigles bein [sic] exactes \& certaines de la prononciation, orthographe, construction \& usage de nostre langue, en faveur des estrenagiers qui en sont desireux, Rouen : Jacques Calloué.

Meigret, L. (1980 [1550]). Le Traité de la grammaire française, in : Le Traité de la grammaire française (1550). Le Menteur de Lucien. Aux lecteurs (1548), édité par Franz Josef Hausmann, Tübingen : Gunter Narr.

Migliorini B. (1944/1945). L'atto di nascita dei vocaboli, Lingua nostra, 6, 6-10.

Möhren, F. (1982). La datation du vocabulaire des imprimés de textes anciens, Revue de Linguistique romane, 46, 328.

Möhren, F. (1986). Lexicologie et lexicographie : points noirs dans la lexicographie des langues romanes, Actes $d u$ Congrès international de linguistique et philologie romanes de Trèves, volume 4, Tubingen : Niemeyer.

Möhren, F. (1988). Principes de rédaction agissant sur l'étymologie : systématique des attestations du Complément de Godefroy et ses matériaux inédits, Travaux de linguistique et de philologie, 26, 185-189.

Möhren, F. (2005). Les limites du projet TLF-Étym : l'avocat du diable a la parole, Actes de la Journée d'étude "Lexicographie historique française : autour de la mise à jour des notices étymologiques du Trésor de la langue française », Nancy: ATILF (CNRS/Université (http://www.atilf.fr/atilf/evenement/JourneeEtude/LHF2005/Stadtler.pdf).

Möhren, F. (2006). L'importance de la critique des sources en étymologie, Actes du Séminaire de méthodologie en étymologie et histoire du lexique, Nancy: ATILF (CNRS/Université Nancy 2/UHP), http://www.atilf.fr/atilf/seminaires/Seminaire Mohren 2005-11.pdf.)

Pfister, M. (1984). Introduzione, LEI ( Lessico etimologico italiano), volume 1, Wiesbaden : Reichert.

Pottier, B. (1958). La valeur de la datation des mots dans la recherche étymologique, Mélanges Wartburg, 581-586.

Richelet, P. (1680). Dictionnaire françois, contenant les mots et les choses, plusieurs nouvelles remarques sur la langue françoise, ses expressions propres, figurées et burlesques, la prononciation des mots les plus difficiles, le genre des noms, le régime des verbes, avec les termes les plus connus des arts et des sciences, Genève : Jean Herman Widerhold.

Schäfer J. (1980). Documentation in the O.E.D. : Shakespeare and Nashe as test cases, Oxford : Clarendon Press.

Städtler, Th. (1988). Zu den Anfängen der französischen Grammatiksprache. Textausgaben und Wortschatzstudien, Tübingen: Niemeyer. 
Städtler, Th. (1999). Témoins précoces de la terminologie grammaticale française, Travaux de linguistique et de philologie, 37, 123-129.

Städtler, Th. (2006). „Auch ist gar keine noth, dasz allen alles verständlich“ (J. Grimm). Observations sur la confection et la présentation des notices étymologiques du Trésor de la langue française informatisé, Actes de la Journée d'étude "Lexicographie historique française: autour de la mise à jour des notices étymologiques du Trésor de la langue française», Nancy: ATILF (CNRS/Université Nancy 2/UHP), (http://www.atilf.fr/atilf/evenement/JourneeEtude/LHF2005/Stadtler.pdf).

Steinfeld N. (2006). Observations méthodologiques sur la traque des premières attestations en étymologie et histoire du lexique (domaine français), Actes du Séminaire de méthodologie en étymologie et histoire du lexique, Nancy, ATILF (CNRS/Université Nancy 2/UHP), (http://www.atilf.fr/atilf/seminaires/Seminaire_Steinfeld_200511.pdf.)

Swiggers P. (1991). L'étymologie (g)allo-romane : perspectives et points de vue, Travaux de linguistique, 23, 97101.

Vaugelas, C. F. (1647). Remarques sur la langue françoise vtiles à ceux qui veulent bien parler et bien escrire, Paris : Veuve J. Camusat et P. Le Petit.

Wartburg von W. (1963 [1946]). Problèmes et méthodes de la linguistique, Paris : PUF.

Zamboni A. (1976). L'Etimologia, Bologna : Zanichelli.

* Mes remerciements les plus chaleureux s'adressent à Éva Buchi (ATILF-CNRS/Nancy Université) et à Thomas Städtler (DEAF, Heidelberg), qui m'ont généreusement fait profiter de leurs réflexions stimulantes sur la lexicologie historique, ainsi qu'à Nadine Steinfeld, Hiltrud Gerner, Pascale Baudinot, Gilles Roques, Jean-Paul Chauveau et Gilles Petrequin (ATILF-CNRS/Nancy Université) qui ont bien voulu assurer une relecture critique du manuscrit.

${ }^{1}$ L'explicitation des sigles utilisés dans le cadre du TLF se fait sur le site http://stella.atilf.fr/tlf.htm

${ }^{2}$ L'explicitation des sigles du TLF-Étym se fait sur le site http://www.atilf.fr/tlf-etym 